\title{
Heads or tails
}

head
regeneration
can be
achieved
by reducing
WNT-
$\beta$-catenin
signalling

Some animals can regenerate organs or entire body parts following injury. A striking example are flatworms, which can develop whole organisms from just a piece of their body. Regeneration occurs via activation of stem cells called neoblasts, which proliferate to form a cell mass (the blastema) that grows and differentiates. However, this regeneration capacity varies between species. Three studies published in Nature now show that in three regeneration-deficient flatworm species - Phagocata kawakatsui, Procotyla fluviatilis and Dendrocoelum lacteum - canonical WNT- $\beta$-catenin signalling prevents the regeneration of heads specifically from tail fragments.

There has been a long-standing debate on whether the capacity of planarian species to reform both heads and tails from any body fragment lies in the existence of two opposing morphogenetic gradients that run along the anterior-posterior axis: one that instructs the formation of head parts and one that instructs the formation of tail parts.

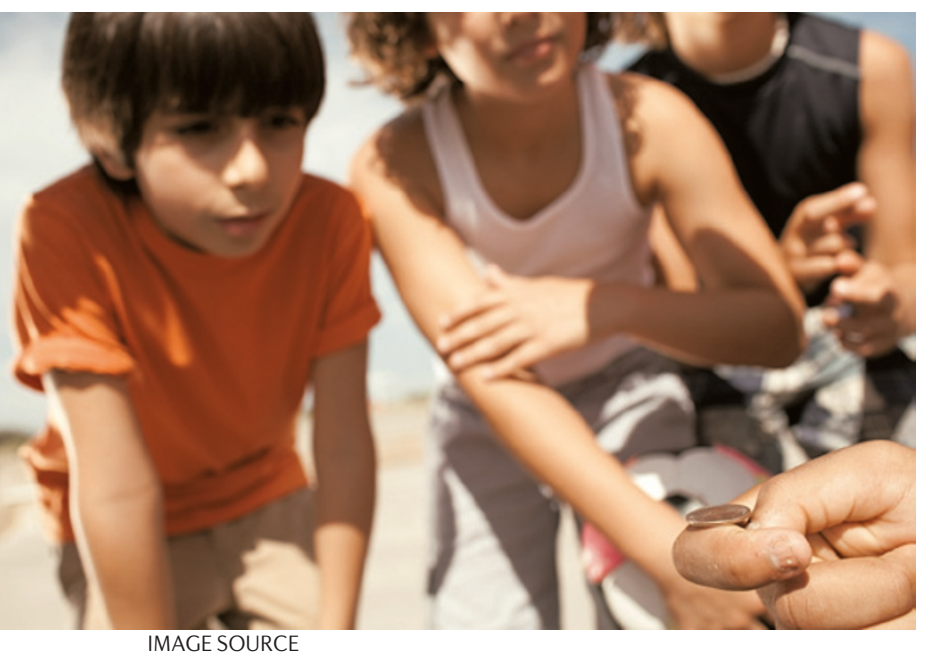

Umesono et al. first validated this hypothesis in Dugesia japonica, which has full regenerative capacity and then tested whether their model explained why P. kawakatsui fails to regenerate heads from tails. It had been previously shown that the WNT- $\beta$-catenin pathway drives the development of tail parts. Here, Umesono et al. find that ERK signalling is required for head regeneration and that ERK activity decreases from head to tail in D. japonica. Furthermore, they show that $\beta$-catenin (the intracellular effector of WNT) interferes with ERK activation, suggesting that ERK and WNT- $\beta$-catenin form two opposing signalling gradients that control anterior-posterior patterning in D. japonica. Importantly, their results also suggest that the formation of head tissues is the 'default' differentiation pathway in regenerative cells in response to ERK activation and support a model in which $P$. kawakatsui fails to regenerate heads from tail fragments because of excessive levels of WNT- $\beta$-catenin signalling in these fragments.

To understand the mechanisms underlying the limited regenerative capacity of $P$. fluviatilis, Sikes and Newmark used next-generation sequencing to identify gene expression differences between regeneration-permissive $\left(\mathrm{Reg}^{+}\right)$tissues and regeneration-deficient $\left(\mathrm{Reg}^{-}\right)$tissues 24 hours after amputation. They found that several genes involved in WNT- $\beta$-catenin signalling were upregulated in amputated Reg- tissues but downregulated in amputated $\mathrm{Reg}^{+}$tissues (compared with intact tissues), suggesting that WNT signals might block regeneration. Indeed, RNAi-mediated disruption of
WNT- $\beta$-catenin signalling resulted in blastema formation and the development of complete heads and brains from Reg- fragments.

Liu et al. observed that, as with P. fluviatilis, RNAi-mediated knockdown of $\beta$-catenin in $D$. lacteum tail pieces rescued regeneration defects and led to the formation of fully functional heads. Furthermore, detailed RNA sequencing timecourse experiments of anterior head-regenerating wound tissues compared with posterior nonregenerating wound tissues revealed that the regeneration defects are not due to failure in blastema formation but rather due to defects in the early stages of head specification. Thus, D. lacteum does not seem to be defective in regeneration per se but is unable to specify the head fate owing to an inability to inhibit WNT signalling in tail pieces.

Together, these studies show that head regeneration can be achieved by reducing WNT- $\beta$-catenin signalling in planarians that normally have regeneration-deficient tails. They also highlight the importance of comparing different species to understand regeneration defects. It will be interesting to study whether the modulation of a single signalling pathway might improve the regenerative capacity in organisms that normally do not regenerate body parts, such as mammals.

Kim Baumann

ORIGINAL RESEARCH PAPERS Umesono, Y. et al. The molecular logic for planarian regeneration along the anterior-posterior axis. Nature $\mathbf{5 0 0}$. 73-76 (2013) |Sikes, J. M. \& Newmark, P. A. Restoration of anterior regeneration in a planarian with limited regenerative ability. Nature 500, 77-80 (2013) | Liu, S.-Y. et al. Reactivating head regrowth in a regeneration-deficient planarian species. Nature 500, 81-84 (2013) 